\title{
Naturally Occurring Canine Invasive Urinary Bladder Cancer: A Complementary Animal Model to Improve the Success Rate in Human Clinical Trials of New Cancer Drugs
}

\author{
Christopher M. Fulkerson, ${ }^{1}$ Deepika Dhawan, ${ }^{1}$ Timothy L. Ratliff, ${ }^{2,3}$ Noah M. Hahn, ${ }^{4}$ and \\ Deborah W. Knapp ${ }^{1,3}$ \\ ${ }^{1}$ Department of Veterinary Clinical Sciences, Purdue University, West Lafayette, IN 47907, USA \\ ${ }^{2}$ Department of Comparative Pathobiology, Purdue University, West Lafayette, IN 47907, USA \\ ${ }^{3}$ Purdue University Center for Cancer Research, Purdue University, West Lafayette, IN 47907, USA \\ ${ }^{4}$ Department Oncology and Urology, Johns Hopkins University School of Medicine and Sidney Kimmel Comprehensive Cancer \\ Center, Baltimore, MD 21287, USA
}

Correspondence should be addressed to Deborah W. Knapp; knappd@purdue.edu

Received 29 December 2016; Accepted 13 March 2017; Published 9 April 2017

Academic Editor: Marco Gerdol

Copyright (c) 2017 Christopher M. Fulkerson et al. This is an open access article distributed under the Creative Commons Attribution License, which permits unrestricted use, distribution, and reproduction in any medium, provided the original work is properly cited.

\begin{abstract}
Genomic analyses are defining numerous new targets for cancer therapy. Therapies aimed at specific genetic and epigenetic targets in cancer cells as well as expanded development of immunotherapies are placing increased demands on animal models. Traditional experimental models do not possess the collective features (cancer heterogeneity, molecular complexity, invasion, metastasis, and immune cell response) critical to predict success or failure of emerging therapies in humans. There is growing evidence, however, that dogs with specific forms of naturally occurring cancer can serve as highly relevant animal models to complement traditional models. Invasive urinary bladder cancer (invasive urothelial carcinoma (InvUC)) in dogs, for example, closely mimics the cancer in humans in pathology, molecular features, biological behavior including sites and frequency of distant metastasis, and response to chemotherapy. Genomic analyses are defining further intriguing similarities between InvUC in dogs and that in humans. Multiple canine clinical trials have been completed, and others are in progress with the aim of translating important findings into humans to increase the success rate of human trials, as well as helping pet dogs. Examples of successful targeted therapy studies and the challenges to be met to fully utilize naturally occurring dog models of cancer will be reviewed.
\end{abstract}

\section{Introduction}

With more than 7.5 million deaths from cancer worldwide each year, there is an ever increasing need to develop better cancer therapies, along with strategies to make cancer care more accessible. Even with tremendous advances in pharmaceutical science and technology, nine out of ten human clinical trials of new cancer therapies fail [1]. One of the major reasons for these failures is the inability of preclinical models to predict the safety and efficacy of new cancer drugs in humans [1]. In in vitro systems, carcinogen-induced, engraftment, and genetically engineered mouse models are instrumental and essential in cancer research [2-7], but they do not possess the collective features (cancer heterogeneity, molecular complexity, invasion, metastasis, and immune cell response) critical to predict success or failure of emerging therapies in humans [1].

The need for relevant cancer models has never been greater. With advances in sequencing methods and genomic analyses, the number of therapeutic candidates with sound biologic rationale justifying investigation in humans continues to grow [8-16]. However, with the rapidly expanding number of combinations of targets and mechanisms of action, the cancer research community has entered an uncharted era in which the number of cancer patients (especially those with uncommon cancer types) is insufficient for 
testing even part of the new therapeutic approaches. Hence, there is an essential need to develop and optimize preclinical animal models to rapidly facilitate more accurate predictors of therapeutic success in approaches ultimately chosen to take forward into humans. With the resurgence of immunotherapies and the understanding of the immune system's role in many types of therapies [16-18], it is critical that animal models also possess a level of immunocompetence similar to that in human cancer patients. As summarized in this review, and using invasive urinary bladder cancer as an example, there is compelling evidence that pet dogs with specific forms of naturally occurring cancer can provide crucially needed relevant animal models to complement other models in preclinical research to help improve the success rate in human clinical trials $[19,20]$.

\section{Challenges in Invasive Urinary Bladder Cancer and a Need for Animal Model Research}

There is a dire need for relevant animal models for research to improve the treatment and management of humans with invasive urinary bladder cancer (InvUC). In humans, InvUC is defined as high-grade UC that has invaded to the depth of the lamina propria (T1 tumors) or beyond (T2-T4 tumors; also commonly called muscle invasive bladder cancer) [21]. Even in patients where the local infiltration is limited to the lamina propria (T1 tumors), that is, not yet into the muscle, InvUC represents an aggressive, often fatal cancer [21]. It should be noted that of all human bladder cancer, up to $80 \%$ of cases are noninvasive, but the invasive cancers represent the most challenging form of the cancer to be treated. Current challenges faced by patients with InvUC in the United States, for example, include the following: (1) 50\% fatality rate and $>16,000$ deaths per year, (2) reduced quality of life from both the cancer and its treatment (cystectomy, radiotherapy, and chemotherapy), and (3) financial costs (\$150,000 median cost of care per patient; $\$ 17$ billion per year lost due to untimely deaths from InvUC) [21-28]. In addition, of the 550,000 people living with nonmuscle invasive cancer in the US, there is a risk of progression to muscle-invasive stages requiring cystectomy in 10-30\% of the patients. The need for better InvUC drugs is crucial as drugs are now being applied in multiple settings including the BCG unresponsive, neoadjuvant and adjuvant perioperative, bladder sparing, and metastatic populations [8, 22-27]. With rare exceptions, relapse after treatment for metastatic disease is inevitable, with few patients achieving durable benefit from the second line treatment [29]. There is, however, hope for vast improvement in the success of InvUC therapy with emerging molecularly targeted drugs, epigenetictargeted drugs, and immunotherapies, provided that animal models can meet research demands [9, 10, 15-18, 25].

Animal models are considered a key to all bladder cancer research, and several instrumental model systems have been defined. Carcinogen-induced tumors in laboratory animals have been used to understand features of bladder tumor development and drug effects at different stages of the process in immunocompetent hosts [2-5]. In syngeneic and immunodeficient mice, tumor cells can be implanted subcutaneously or orthotopically in the bladder to evaluate drug activity. Different types of cells including tumor cells, epithelial cells, stromal cells, and embryonic urogenital sinus mesenchymal cells can be implanted under the renal capsule to evaluate drugs and to study epithelial-stromal interactions [2, 3, 30]. Genetically engineered mouse models (GEMs) have been widely used in other types of cancer to analyze tumor phenotype, to study some subtypes, to investigate candidate genes and signaling pathways, and to test drugs, and GEMs are beginning to be used more in bladder cancer research [2-4]. GEMs for bladder tumors include those with altered $p R B$ and/or $p 53[31,32]$; Pten with or without p53 [33, 34]; Kras and Hras [35, 36]; Egfr [37]; p21 [35]; IL17, IL12, IL23, and IFNY [38-40]; and others [2-4]. Patient-derived xenograft models are also promising tools to study drug effects on an individual's tumor, and these are now being described for bladder cancer [41, 42]. As with other cancers, however, these animal models of InvUC do not meet the increasing demands of the complex cancer therapies being developed to treat the aggressive heterogenous cancer in humans [8-18, 43-52]. But there is growing evidence that an intriguing complementary animal model, naturally occurring InvUC in dogs, could help address these demands $[19,53,54]$.

\section{Naturally Occurring Canine Invasive Bladder Cancer and Relevance to Human Invasive Bladder Cancer}

The vast majority of naturally occurring bladder cancer in dogs consists of InvUC [19]. It should be noted that the nomenclature for the $\mathrm{T}$ stages for canine bladder cancer differs somewhat from that used in humans [19]. In dogs, "T1 tumors" are superficial and have not invaded the lamina propria as T1 tumors in humans have. Therefore, in dogs, invasive cancer is categorized as T2 or T3. There is another difference in the staging system between dogs and humans. In dogs, T2 tumors are any tumors that have invaded the muscularis, while T3 tumors are those that have invaded neighboring organs; thus, T2 and T3 tumors comprise the invasive UC reported here. In humans, T2 tumors have invaded the muscle, T3 tumors have invaded the deep fat under the muscle, and T4 tumors have invaded the neighboring organs. Nonmuscle invasive bladder cancer, which is very common in people with bladder cancer, is rare in dogs [19]. Canine bladder cancer offers a model of the more lethal bladder cancer biology in humans, InvUC. Canine InvUC mimics human InvUC in the following: (1) pathology including cellular features, tumor heterogeneity, and infiltrating immune cells [19, 20, 55-62]; (2) local invasion and patterns of distant metastases (lungs and other organs) in $>50 \%$ of individuals [19]; and (3) response to cisplatin [63-65], carboplatin [66], vinblastine [67, 68], doxorubicin [69], and gemcitabine [70] (summarized in Table 1). Further similarities between canine and human InvUC include shared druggable mutations and pathway variants, epigenetic targets, and evidence for gene 
TABLE 1: Similarities and differences in naturally occurring invasive urothelial carcinoma between dogs and humans.

\begin{tabular}{l}
\hline Similarities between dogs and humans \\
Physiological age of onset and clinical symptoms \\
Pathologically high grade, heterogenous cancer \\
Molecular subtypes (e.g., luminal, basal) \\
Epigenetic features \\
Shared molecular targets (e.g., EGFR, CDKN2B, PIK3CA, BRCA2, and NFkB) \\
Local cancer invasion into the bladder wall \\
Distant cancer metastases in $\geq 50 \%$ of subjects \\
Response to chemotherapy (e.g., cisplatin, carboplatin, and vinblastine) \\
Differences between dogs and humans \\
Sex differences (male: female ratio $2: 1$ in humans, $0.5: 1$ in dogs; although most dogs studied had been spayed or neutered) \\
Tumor location in bladder (more often trigonal in dogs; more variable in humans) \\
Dog tumors possess dog homologue of BRAF V600E mutation common in human melanoma (human InvUC has other variants in \\
MAPK signaling)
\end{tabular}

patterns of molecular subtypes (basal and luminal) [71-78]. In microarray analyses of genes differentially expressed between canine bladder with InvUC and normal canine bladder, two distinct clusters were identified in the InvUC samples [71]. A recent reanalysis of the data comparing findings to a list of $>600$ genes that segregate human InvUC into luminal and basal subtypes demonstrated that these two clusters in canine InvUC align closely with luminal and basal expression patterns in humans. Pertinent to in vitro studies, the establishment of canine InvUC cell lines has been reported [79, 80].

Although the molecular characterization of canine InvUC is in the early stages, intriguing similarities to human InvUC have been noted. Mutations in several genes implicated in human InvUC (EGFR [9, 50, 81], CDKN2B [82, 83], PIK3CA [9, 82], BRCA2 [84], and NFkB [85]) have been identified in dogs [72]. EGFR overexpression, a particularly interesting target, has been confirmed by immunohistochemistry (IHC) in $73 \%$ of canine InvUC cases, similar to that in humans, and a canine trial of an EGFR-targeted compound is ongoing $[71,81]$. The expression of estrogen and androgen receptors has also been reported in canine InvUC [19]. In addition, in microarray studies (canine normal bladder, canine InvUC, and human microarray data-GEO database), there were $>400$ genes differentially expressed between normal and tumor in both dogs and humans [71, 73].

Although there are marked similarities between canine and human InvUC, expected differences do occur (Table 1). The male: female ratio is $\sim 2: 1$ in humans with InvUC [21] but $\sim 0.5: 1$ in dogs affected by InvUC [19], although most dogs studied were spayed or neutered. In an intriguing molecular difference between canine and human InvUC, the majority of canine InvUCs carry a mutation in the MAPK signaling pathway (dog homologue of $B R A F^{\mathrm{V} 600 \mathrm{E}}$ ) reported in several human cancers $[72,86,87]$. While $B R A F$ mutations are rare in human InvUC, other activating mutations in the MAPK pathway occur in $\sim 30 \%$ of cases [9]. The finding of the $B R A F$ mutation in canine InvUC opens the door for research in dogs with bladder cancer to be applied across many types of human cancer.
Canine clinical trials of new cancer drugs in which dogs continue life as pets while participating in a trial are well accepted and are considered a win-win scenario that benefits each participating dog and generates knowledge to help people and pet dogs $[19,88,89]$. Although InvUC only comprises $2 \%$ of all dog cancers, approximately six million new canine cancer cases are diagnosed yearly in the US, resulting in an ample number of dogs with InvUC available for translational research [90]. A compelling question of any model is the extent to which drug effects in the model will predict drug effects in humans. While this proof-of-concept work has been limited to date, our group has published an unexpected beneficial drug effect first identified in dogs with InvUC that was then found in an exploratory human trial. Briefly, cyclooxygenase (COX) inhibitors have had intriguing antitumor effects and chemotherapy-enhancing effects on dogs with InvUC [19, 63, 65, 68, 91-93]. Given as single agents, COX inhibitors induce remission in $18-20 \%$ of dogs with InvUC and cause tumor stabilization in approximately $50-55 \%$ of dogs $[19,91]$. The addition of COX inhibitors substantially enhances the remission rate of cisplatin (20\% with the single agent cisplatin, 50-70\% with combined drugs) $[63,65,92]$ and vinblastine (23\% with the single agent vinblastine, $58 \%$ with combined drugs) [68]. In a neoadjuvant trial of a COX-2 inhibitor, celecoxib, given between cystoscopic diagnosis and cystectomy in humans, the same biological effects associated with COX inhibitor-induced remission in dogs were found in humans receiving celecoxib [94].

Another aspect of InvUC in dogs which offers key opportunities for translational research, as well as management of the cancer in dogs, is the very strong dog breed-associated risk. Scottish Terriers have an 18-20-fold increased risk for developing InvUC compared to mixed breed dogs, and Eskimo Dogs, Shetland Sheepdogs, West Highland White Terriers, Keeshonds, Samoyeds, and Beagles have a 3-6fold increased risk [19]. This offers an unparalleled setting to study heritable risk and gene-environment interactions leading to InvUC and to study early intervention strategies [19, 20, 76, 95-97]. In Scottish Terriers, dogs exposed to lawn chemicals had a 7 -fold higher risk of bladder cancer than 
those not exposed [95]. In humans, approximately half of bladder cancer is due to first-hand cigarette smoke [21]. In dogs across all breeds, a link to second-hand smoke has not been proven, but studies in specific high-risk breeds are indicated. Although heritable factors are thought to play an important role in InvUC risk in humans, groups of humans with this level of heritable risk for the cancer have not been identified due to the tremendous genetic diversity in humans. Once heritable factors are identified in dogs, those same factors or related factors and mechanisms could be investigated in humans [98]. Of equal importance, the strong dog breedassociated risk for InvUC is defining groups of dogs in which to study screening protocols, methods for early detection, and early intervention strategies with promising work ongoing $[19,20,76,95-98]$.

\section{Examples of Targeted Therapy Research Utilizing the Canine Bladder Cancer Model}

4.1. Folate-Targeted Therapy. There are published examples of studies in dogs with InvUC which are aimed at translating into humans. One example involves folate-targeted therapy, an approach which stems from the finding that there is much higher uptake of folate (vitamin B9) and folate drug conjugates in certain cancers than in normal tissues [99]. Upregulation of folate receptors (FRs), especially $\mathrm{FR} \alpha$, has been noted in several forms of human cancer, while the expression of similar FRs in normal tissues is limited [99, 100]. Although folate-targeted therapy has been evaluated in humans with ovarian cancer and other solid tumors [101, 102], this therapeutic approach has not yet been studied in human bladder cancer. To determine the potential value of folate-targeted therapy in human InvUC, research was conducted to determine FR expression in the human cancer and in the canine InvUC model and the safety and activity of folate-targeted therapy in dogs with InvUC. In dogs, FR expression was detected in $78 \%$ of primary InvUC and $80 \%$ of nodal metastases by IHC (PU17, polyclonal antibody, Endocyte, West Lafayette, IN) [103]. Scintigraphy was used to confirm folate uptake in primary and metastatic lesions of dogs with InvUC. FR expression was also detected in human InvUC, although further work is required to determine the frequency of the expression. The FR expression in human InvUC was similar to that in dogs when using the PU17 antibody for IHC, but immunoreactivity to a human monoclonal antibody (mab343) was less frequent [103].

A dose escalation study of folate-targeted vinblastine (EC0905, Endocyte) was conducted in dogs with biopsy-confirmed, FR-positive InvUC [103]. As in all clinical trials in dogs at our institution, the study was approved by the Purdue Animal Care and Use Committee, and informed pet owner consent in writing was required for all participating dogs. The maximum tolerated dose (MTD) of EC0905 in dogs $(0.25 \mathrm{mg} / \mathrm{kg}$ IV weekly) was determined with neutropenia occurring at higher doses. With informative laboratory dog work done before the pet dog trial, only 10 pet dogs with InvUC were required to establish the MTD. In 10 dogs, the tumor responses included partial remission $(\geq 50 \%$ reduction in tumor volume) in 5 dogs and stable disease $(<50 \%$ change in tumor volume) in 4 dogs. The tumor response could not be determined in one dog. With these initial promising findings, a more extensive trial of folate-targeted therapy in dogs with InvUC is ongoing. Demonstrating the benefit in dogs could offer the justification to expand the human application of folate-targeted therapy to bladder cancer, as well as the other cancers currently being investigated.

\subsection{Epigenetic-Based Therapies-Demethylating Agent} Trials. Another example of targeted therapy research in canine bladder cancer with a translational goal involves drugs aimed at epigenetic changes that lead to cancer development and progression in the absence of DNA mutations [104108]. One of the key epigenetic events is the aberrant methylation in the promoter region of tumor suppressor genes, resulting in gene silencing. Aberrant DNA methylation has been identified in multiple genes in human InvUC [107, 108]. DNA methyltransferase 1 (DNMT1), which is a key player in the aberrant methylation process, has been noted to be overexpressed in human and canine InvUC [73, 109]. When considering the design of clinical trials of demethylating agents in humans, key valuable information that could be rapidly obtained from dogs includes efficacy, safety, and treatment scheduling. With the need for this information, a clinical trial of the demethylating agent, 5-azacitidine (5AzaC), in dogs with InvUC was conducted [74]. Doses were escalated in 2 different dose schedules (daily treatment for 5 sequential days for one cycle per month or daily treatment for 5 sequential days for two cycles per month). Of 18 dogs evaluable for tumor response, partial remission, stable disease, and progressive disease were observed in 4 (22.2\%), 9 (50.0\%), and $4(22.2 \%)$ dogs, respectively. Although remission is certainly preferred, durable stable disease (i.e., cancer control) of bladder cancer can also be a very beneficial response. The MTD in each treatment schedule was defined, with neutropenia occurring at higher doses. Consistent 5AzaC urine levels $(205-857 \mathrm{ng} / \mathrm{ml})$ were measured.

While 5-AzaC had promising activity in dogs with InvUC, the route of administration (subcutaneous injection) required in-hospital treatment. Zebularine, an orally bioavailable cytidine analog with demethylating activity that has been extensively studied in vitro, circumvents the need for an intermittent in-hospital dosing schedule and allows for convenient daily dosing. The ease of use and flexible dose scheduling of an orally bioavailable demethylating agent offers advantages over injectable agents; thus, zebularine was investigated in laboratory dogs and tumor-bearing dogs [98]. Based on extrapolation from in vitro data and data in rodents, laboratory dogs were initially treated with $8 \mathrm{mg} / \mathrm{kg}$ to determine plasma pharmacokinetics and then daily with $4 \mathrm{mg} / \mathrm{kg}$ to determine toxicity. Interestingly, and unexpectedly based on previously published in vitro and in vivo rodent data, daily treatment with $4 \mathrm{mg} / \mathrm{kg}$ zebularine resulted in severe neutropenia that was resolved with discontinuation of the drug, but required supportive care. Maximum plasma concentrations following treatment with $8 \mathrm{mg} / \mathrm{kg}$ and $4 \mathrm{mg} / \mathrm{kg}$ were $23 \pm 4.8 \mu \mathrm{M}$ and $8.6 \pm 1.4 \mu \mathrm{M}$, respectively, which were much lower than concentrations previously reported in vitro and in vivo. Neutropenia was not observed 
in a cohort of three tumor-bearing dogs treated with $4 \mathrm{mg} / \mathrm{kg}$ once every 21 days, including two dogs with InvUC.

The study of zebularine in laboratory dogs and tumorbearing dogs demonstrates the utility of studies in a canine model, as it was quickly established that zebularine concentrations previously achieved in vitro and in vivo in rodents could not be achieved safely in a veterinary clinical setting in dogs. However, two dogs with InvUC experienced stable disease for 12 and 18 weeks while receiving treatment with zebularine ( $4 \mathrm{mg} / \mathrm{kg}$ q21 days), suggesting a clinical benefit even at doses much lower than those previously reported in vitro and in vivo in rodents and primates [109]. A subsequent dose escalation trial in dogs with InvUC is in progress with the aim of defining a tolerable, long-term daily treatment schedule, with promising results to date. These studies demonstrate the promise for demethylating treatment strategies in InvUC as well as findings in dogs that would be expected to be translated into beneficial effects in humans and inform the design of human clinical trials.

\section{Challenges to Be Met in Applying Naturally Occurring Canine Cancer Models}

There is compelling evidence for the value of studies of pet dogs with naturally occurring cancer as a complementary animal model for improving the success rate in human trials, thus helping reduce the morbidity, mortality, and health care cost associated with cancer treatment, as well as greatly reducing the overall cost of the drug development process. There are, of course, challenges to be met in applying pet dog models. First, the characterization of canine cancer at the molecular level is in the early stages. While it is further along in some cancers, such as InvUC [19, 58, 71-73, 75, 110], than in others, there is still much work to be done. Second, the "canine model system" has not yet been fully tested to prove that the outcome in dog studies will predict the outcome in human studies. There is early evidence of this [88, 94], and more work is in progress, but, currently without this proof, major pharmaceutical companies have hesitated to invest in canine studies. And, without the funding, the proof of concept work in dogs cannot be done. An appropriately powered, randomized three-arm treatment trial in dogs, depending on the agent being tested and numbers of dogs needed, would cost a fraction of a similar human trial, but would cost much more than a laboratory rodent study. In addition, clinical trials in dogs can take a few months to two to three years, while the rodent studies are accomplished more quickly. Third, the number of veterinary clinician scientists and programs with the requisite skills, knowledge in comparative oncology, and specific tumor caseload would be required to grow should the demand for comparative oncology trials escalate. In addition to academic programs, there are other programs in place to facilitate canine clinical trials including the Comparative Oncology Trials Consortium directed by the Comparative Oncology Program at the National Cancer Institute [111]. Another consortium, the Canine Comparative Oncology Genomics Consortium (CCOGC) [112], sponsored by the National Cancer Institute, the American Kennel Club Canine Health Foundation, the
Morris Animal Foundation, and Pfizer, is facilitating access to tumor and normal tissues and other samples from dogs through a biospecimen repository.

With the tremendous benefits to be gained from the full utilization of naturally occurring canine animal models of cancer, the challenges described above can certainly be met with a concerted effort in this area. As therapeutic drugs continue to become specifically targeted or engaged in complex interactions with the intact immune system, naturally occurring canine models that replicate key features of human cancer (cancer heterogeneity, molecular complexity, invasion, metastasis, and immune cell response) are uniquely positioned for the investigation of new therapeutic drugs and to complement work in traditional animal models. With the reports of immune checkpoints in canine tumors that are critical in human cancer, there is considerable interest in developing canine-specific therapeutic antibodies to block these checkpoints $[62,113-116]$. The promise of comparative oncology research to improve the success of human clinical trials has never been more important.

\section{Conflicts of Interest}

The authors declare that there is no conflict of interests regarding the publication of this paper.

\section{Acknowledgments}

The authors thank the staff and doctors in the Purdue Comparative Oncology Program for all of their work in the studies summarized here. The authors also thank the many dedicated pet dog owners who allowed their dogs to participate in clinical research for providing support for bladder cancer research at Purdue University.

\section{References}

[1] I. W. Mak, N. Evaniew, and M. Ghert, "Lost in translation: animal models and clinical trials in cancer treatment," American Journal of Translational Research, vol. 6, no. 2, pp. 114-118, 2014.

[2] N. Zhang, D. Li, J. Shao, and X. Wang, "Animal models for bladder cancer: the model establishment and evaluation," Oncology Letters, vol. 9, no. 4, pp. 1515-1519, 2015.

[3] T. Kobayashi, T. B. Owczarek, J. M. McKiernan, and C. Abate-Shen, "Modelling bladder cancer in mice: opportunities and challenges," Nature Reviews. Cancer, vol. 15, no. 1, pp. 42-54, 2015.

[4] I. Ahmad, O. J. Sansom, and H. Y. Leung, "Exploring molecular genetics of bladder cancer: lessons learned from mouse models," Disease Models \& Mechanisms, vol. 5, no. 3, pp. 323-332, 2012.

[5] Z. He, W. Kosinska, Z. L. Zhao, X. R. Wu, and J. B. Guttenplan, "Tissue-specific mutagenesis by N-butyl-N(4-hydroxybutyl) nitrosamine as the basis for urothelial carcinogenesis," Mutation Research, vol. 742, no. 1-2, pp. 92-95, 2012.

[6] D. P. Vang, G. T. Wurz, S. M. Griffey et al., "Induction of invasive transitional cell bladder carcinoma in immune intact human MUC1 transgenic mice: a model for immunotherapy 
development," Journal of Visualized Experiments, vol. 80, article e50868, 2013.

[7] J. M. Levitt, W. Jian, S. P. Lerner, and G. Sonpavde, "A conventional preclinical schedule of cisplatin is more effective than a metronomic frequent bolus schedule for urothelial carcinoma," Urologic Oncology, vol. 31, no. 2, pp. 234-240, 2013.

[8] M. Rouanne, Y. Loriot, T. Lebret, and J. C. Soria, "Novel therapeutic targets in advanced urothelial carcinoma," Critical Reviews in Oncology/Hematology, vol. 98, pp. 106-115, 2016.

[9] Cancer Genome Atlas Research Network, "Comprehensive molecular characterization of urothelial bladder carcinoma," Nature, vol. 507, no. 7492, pp. 315-322, 2014.

[10] G. Iyer, H. Al-Ahmadie, and N. Schultz, "Prevalence and co-occurrence of actionable genomic alterations in highgrade bladder cancer," Journal of Clinical Oncology, vol. 31, no. 25, pp. 3133-3140, 2013.

[11] J. Kim, R. Akbani, C. J. Creighton et al., "Invasive bladder cancer: genomic insights and therapeutic promise," Clinical Cancer Research, vol. 21, no. 20, pp. 4514-4524, 2015.

[12] K. E. M. van Kessel, T. C. M. Zuiverloon, A. R. Alberts, J. L. Boormans, and E. C. Zwarthoff, "Targeted therapies in bladder cancer: an overview of in vivo research," Nature Reviews. Urology, vol. 12, no. 12, pp. 681-694, 2015.

[13] W. A. Schulz, E. A. Koutsogiannouli, G. Niegisch, and M. J. Hoffmann, "Epigenetics of urothelial carcinoma," Methods in Molecular Biology, vol. 1238, pp. 183-215, 2015.

[14] I. Ibragimova, E. Dulaimi, M. J. Slifker, D. D. Chen, R. G. Uzzo, and P. Cairns, "A global profile of gene promoter methylation in treatment-naïve urothelial cancer," Epigenetics, vol. 9, no. 5, pp. 760-773, 2014.

[15] A. Besaratinia, M. Cockburn, and S. Tommasi, "Alterations of DNA methylome in human bladder cancer," Epigenetics, vol. 8, no. 10, pp. 1013-1022, 2013.

[16] D. M. Pardoll, "The blockade of immune checkpoints in cancer immunotherapy," Nature Reviews. Cancer, vol. 12, no. 4, pp. 252-264, 2012.

[17] T. Powles, J. P. Eder, G. D. Fine et al., "MPDL3280A (antiPD-L1) treatment leads to clinical activity in metastatic bladder cancer," Nature, vol. 515, no. 7528, pp. 558-562, 2014.

[18] J. E. Rosenberg, J. Hoffman-Censits, T. Powles et al., "Atezolizumab in patients with locally advanced and metastatic urothelial carcinoma who have progressed following treatment with platinum-based chemotherapy: a single-arm, multicentre, phase 2 trial," Lancet, vol. 387, no. 10031, pp. 1909-1920, 2016.

[19] D. W. Knapp, J. A. Ramos-Vara, G. E. Moore, D. Dhawan, P. L. Bonney, and K. E. Young, "Urinary bladder cancer in dogs, a naturally occurring model for cancer biology and drug development," ILAR Journal, vol. 55, no. 1, pp. 100-118, 2014.

[20] D. W. Knapp, D. Dhawan, and E. Ostrander, "Lassie, Toto, and fellow pet dogs: poised to lead the way for advances in cancer prevention," American Society of Clinical Oncology Educational Book, pp. e667-e672, 2015.

[21] B. Czerniak, C. Dinney, and D. McConkey, "Origins of bladder cancer," Annual Review of Pathology, vol. s23, no. 11, pp. 149-174, 2016.

[22] R. Chou, S. S. Selph, D. I. Buckley et al., "Treatment of muscle-invasive bladder cancer: a systematic review," Cancer, vol. 122, no. 6, pp. 842-851, 2015.
[23] E. M. Carballido and J. E. Rosenberg, "Optimal treatment for metastatic bladder cancer," Current Oncology Reports, vol. 16, no. 9, p. 404, 2014.

[24] G. Arcangeli, L. Strigari, and S. Arcangeli, "Radical cystectomy versus organ-sparing trimodality treatment in muscleinvasive bladder cancer: a systematic review of clinical trials," Critical Reviews in Oncology/Hematology, vol. 95, no. 3, pp. 87-396, 2015.

[25] J. C. Park and N. M. Hahn, "Bladder cancer: a disease ripe for major advances," Clinical Advances in Hematology \& Oncology, vol. 12, no. 2, pp. 838-845, 2014.

[26] T. L. Rose and M. I. Milowsky, "Improving systemic chemotherapy for bladder cancer," Current Oncology Reports, vol. 18, no. 5, p. 27, 2016.

[27] G. Niegisch, M. Retz, R. Siener, and P. Albers, "Quality of life in patients with cisplatin-resistant urothelial cancer: typical ailments and effect of paclitaxel-based salvage therapy," Urologic Oncology, vol. 34, no. 6, article e15-21, p. 256, 2016.

[28] R. S. Svatek, B. K. Hollenbeck, S. Holmäng et al., "The economics of bladder cancer: costs and considerations of caring for this disease," European Urology, vol. 66, no. 2, pp. 253-262, 2014

[29] J. Bellmunt, C. Théodore, T. Demkov et al., "Phase III trial of vinflunine plus best supportive care compared with best supportive care alone after a platinum-containing regimen in patients with advanced transitional cell carcinoma of the urothelial tract," Journal of Clinical Oncology, vol. 27, no. 27, pp. 4454-4461, 2009.

[30] M. El Behi, S. Krumeich, C. Lodillinsky et al., "An essential role for decorin in bladder cancer invasiveness," $E M B O$ Molecular Medicine, vol. 5, no. 12, pp. 1835-1851, 2013.

[31] F. He, M. Lan, X. Zheng et al., "Deficiency of pRb family proteins and p53 in invasive urothelial tumorigenesis," Cancer Research, vol. 69, no. 24, pp. 9413-9421, 2009.

[32] M. Santos, M. Martínez-Fernández, M. Dueñas et al., "In vivo disruption of an Rb-E2F-Ezh2 signaling loop causes bladder cancer," Cancer Research, vol. 74, no. 22, pp. 6565-6577, 2014.

[33] X. Yang, L. G. Francisco, E. E. Genova et al., "Simultaneous activation of Kras and inactivation of p53 induces soft tissue sarcoma and bladder urothelial hyperplasia," PloS One, vol. 8, no. 9, article e74809, 2013.

[34] I. Ahmad, J. P. Morton, L. B. Singh et al., “ $\beta$-Catenin activation synergizes with PTEN loss to cause bladder cancer formation," Oncogene, vol. 30, no. 2, pp. 178-189, 2011.

[35] I. Ahmad, R. Patel, Y. Liu et al., "Ras mutation cooperates with $\beta$-catenin activation to drive bladder tumourigenesis," Cell Death \& Disease, vol. 2, no. 3, article e124, 2011.

[36] X.-R. Wu, "Urothelial tumorigenesis: a tale of divergent pathways," Nature Reviews. Cancer, vol. 5, no. 9, pp. 713-725, 2005.

[37] J. Cheng, H. Huang, Z.-T. Zhang et al., "Overexpression of epidermal growth factor receptor in urothelium elicits urothelial hyperplasia and promotes bladder tumor growth," Cancer Research, vol. 62, no. 14, pp. 4157-4163, 2002.

[38] M. Kortylewski, H. Xin, M. Kujawski et al., "Regulation of the IL-23 and IL-12 balance by Stat3 signaling in the tumor microenvironment," Cancer Cell, vol. 15, no. 2, pp. 114-123, 2009.

[39] L. Wang, T. Yi, M. Kortylewski, D. M. Pardoll, D. Zeng, and H. Yu, "IL-17 can promote tumor growth through an IL-6- 
Stat3 signaling pathway," The Journal of Experimental Medicine, vol. 206, no. 7, pp. 1457-1464, 2009.

[40] J. L. Langowski, X. Zhang, L. Wu et al., "IL-23 promotes tumour incidence and growth," Nature, vol. 442, no. 7101, pp. 461-465, 2006.

[41] W. Jäger, H. Xue, T. Hayashi et al., "Patient-derived bladder cancer xenografts in the preclinical development of novel targeted therapies," Oncotarget, vol. 6, no. 25, pp. 21522-21532, 2015.

[42] C. X. Pan, H. Zhang, C. G. Tepper et al., "Development and characterization of bladder cancer patient-derived xenografts for molecularly guided targeted therapy," PloS One, vol. 10, no. 8, article e0134346, 2015.

[43] D. J. McConkey, W. Choi, A. Ochoa, A. Siefker-Radtke, B. Czerniak, and C. P. Dinney, "Therapeutic opportunities in the intrinsic subtypes of muscle-invasive bladder cancer," Hematology/Oncology Clinics of North America, vol. 29, no. 2, pp. 377-394, 2015.

[44] G. Sjödahl, M. Lauss, K. Lövgren et al., "A molecular taxonomy for urothelial carcinoma," Clinical Cancer Research, vol. 18, no. 12, pp. 3377-3386, 2012.

[45] D. Lindgren, A. Frigyesi, S. Gudjonsson et al., "Combined gene expression and genomic profiling define two intrinsic molecular subtypes of urothelial carcinoma and gene signatures for molecular grading and outcome," Cancer Research, vol. 70, no. 9, pp. 3463-3472, 2010.

[46] W. Choi, B. Czerniak, A. Ochoa et al., "Intrinsic basal and luminal subtypes of muscle-invasive bladder cancer," Nature Reviews. Urology, vol. 11, no. 7, pp. 400-410, 2014.

[47] D. J. McConkey, W. Choi, and C. P. N. Dinney, "New insights into subtypes of invasive bladder cancer: considerations of the clinician," European Urology, vol. 66, no. 4, pp. 609610, 2014.

[48] W. Choi, S. Porten, S. Kim et al., "Identification of distinct basal and luminal subtypes of muscle-invasive bladder cancer with different sensitivities to frontline chemotherapy," Cancer Cell, vol. 25, no. 2, pp. 152-165, 2014.

[49] J. S. Damrauer, K. A. Hoadley, D. D. Chism et al., "Intrinsic subtypes of high-grade bladder cancer reflect the hallmarks of breast cancer biology," Proceedings of the National Academy of Sciences of the United States of America, vol. 111, no. 8, pp. 3110-3115, 2014.

[50] S. Rebouissou, I. Bernard-Pierrot, A. de Reyniès et al., "EGFR as a potential therapeutic target for a subset of muscle-invasive bladder cancers presenting a basal-like phenotype," Science Translational Medicine, vol. 6, no. 244, p. 244ra91, 2014.

[51] G. Guo, X. Sun, C. Chen et al., "Whole-genome and whole-exome sequencing of bladder cancer identifies frequent alterations in genes involved in sister chromatid cohesion and segregation," Nature Genetics, vol. 45, no. 12, pp. 459-1463, 2013.

[52] Q. Q. Li, J. J. Hao, Z. Zhang et al., "Histone deacetylase inhibitor-induced cell death in bladder cancer is associated with chromatin modification and modifying protein expression: a proteomic approach," International Journal of Oncology, vol. 48, no. 6, pp. 2591-2607, 2016.

[53] J. D. Schiffman and M. Breen, "Comparative oncology: what dogs and other species can teach us about humans with cancer," Philosophical Transactions of the Royal Society B: Biological Sciences, vol. 370, no. 1673, p. 20140231, 2015.
[54] C. E. Alvarez, "Naturally occurring cancers in dogs: insights for translational genetics and medicine," ILAR Journal, vol. 55, no. 1, pp. 16-45, 2014.

[55] V. E. Valli, A. Norris, R. M. Jacobs et al., "Pathology of canine bladder and urethral cancer and correlation with tumour progression and survival," Journal of Comparative Pathology, vol. 113, no. 2, pp. 113-130, 1995.

[56] D. J. Patrick, S. D. Fitzgerald, I. A. Sesterhenn, C. J. Davis, and M. Kiupel, "Classification of canine urinary bladder urothelial tumours based on the World Health Organization/International Society of Urological Pathology consensus classification," Journal of Comparative Pathology, vol. 135, no. 4, pp. 190-199, 2006.

[57] M. Cekanova, M. J. Uddin, J. W. Bartges et al., "Molecular imaging of cyclooxygenase- 2 in canine transitional cell carcinomas in vitro and in vivo," Cancer Prevention Research (Philadelphia, Pa.), vol. 6, no. 5, pp. 466-476, 2013.

[58] S. G. Shapiro, S. Raghunath, C. Williams et al., "Canine urothelial carcinoma: genomically aberrant and comparatively relevant," Chromosome Research, vol. 23, no. 2, pp. 311-331, 2015.

[59] S. I. Mohammed, D. W. Knapp, D. G. Bostwick et al., "Expression of cyclooxygenase-2 (COX-2) in human invasive transitional cell carcinoma (TCC) of the urinary bladder," Cancer Research, vol. 59, no. 22, pp. 5647-5650, 1999.

[60] D. W. Knapp, N. W. Glickman, D. B. Denicola, P. L. Bonney, T. L. Lin, and L. T. Glickman, "Naturally-occurring canine transitional cell carcinoma of the urinary bladder A relevant model of human invasive bladder cancer," Urologic Oncology, vol. 5, no. 2, pp. 47-59, 2000.

[61] G. Hartley, E. Faulhaber, A. Caldwell et al., "Immune regulation of canine tumour and macrophage PD-L1 expression," Veterinary and Comparative Oncology, 2016, Epub ahead of print.

[62] N. Maekawa, S. Konnai, R. Ikebuchi et al., "Expression of PD-L1 on canine tumor cells and enhancement of IFN- $\gamma$ production from tumor-infiltrating cells by PD-L1 blockade," PloS One, vol. 9, no. 6, article e98415, 2014.

[63] D. W. Knapp, G. W. Glickman, W. R. Widmer et al., "Cisplatin versus cisplatin combined with piroxicam in a canine model of human invasive urinary bladder cancer," Cancer Chemotherapy and Pharmacology, vol. 46, no. 3, pp. 221-226, 2000.

[64] R. Chun, D. W. Knapp, and W. R. Widmer, "Cisplatin treatment of transitional cell carcinoma of the urinary bladder in dogs: 18 cases (1983-1993)," Journal of the American Veterinary Medical Association, vol. 209, no. 9, pp. 1588-1591, 1996.

[65] D. W. Knapp, C. J. Henry, W. R. Widmer et al., "Randomized trial of cisplatin versus firocoxib versus cisplatin/firocoxib in dogs with transitional cell carcinoma of the urinary bladder," Journal of Veterinary Internal Medicine, vol. 27, no. 1, pp. 126-133, 2013.

[66] P. A. Boria, N. W. Glickman, B. R. Schmidt et al., "Carboplatin and piroxicam therapy in 31 dogs with transitional cell carcinoma of the urinary bladder," Veterinary and Comparative Oncology, vol. 3, no. 2, pp. 73-80, 2005.

[67] E. J. Arnold, M. O. Childress, L. M. Fourez et al., "Clinical trial of vinblastine in dogs with transitional cell carcinoma of the urinary bladder," Journal of Veterinary Internal Medicine, vol. 25, no. 6, pp. 1385-1390, 2011. 
[68] D. W. Knapp, A. Ruple-Czerniak, J. A. Ramos-Vara, J. F. Naughton, C. M. Fulkerson, and S. I. Honkisz, "A nonselective cyclooxygenase inhibitor enhances the activity of vinblastine in a naturally-occurring canine model of invasive urothelial carcinoma," Bladder Cancer, vol. 2, no. 2, pp. 241-250, 2016.

[69] C. Robat, J. Burton, D. Thamm, and D. Vail, "Retrospective evaluation of doxorubicin-piroxicam combination for the treatment of transitional cell carcinoma in dogs," The Journal of Small Animal Practice, vol. 54, no. 2, pp. 67-74, 2013.

[70] L. Marconato, E. Zini, D. Lindner, L. Suslak-Brown, V. Nelson, and A. K. Jeglum, "Toxic effects and antitumor response of gemcitabine in combination with piroxicam treatment in dogs with transitional cell carcinoma of the urinary bladder," Journal of the American Veterinary Medical Association, vol. 238, no. 8, pp. 1004-1010, 2011.

[71] D. Dhawan, M. Paoloni, S. Shukradas et al., "Comparative gene expression analyses identify luminal and basal subtypes of canine invasive urothelial carcinoma that mimic patterns in human invasive bladder cancer," PloS One, vol. 10, no. 9, article e0136688, 2015.

[72] B. Decker, H. G. Parker, D. Dhawan et al., "Homologous mutation to human BRAF V600E is common in naturally occurring canine bladder cancer-evidence for a relevant model system and urine-based diagnostic test," Molecular Cancer Research, vol. 13, no. 6, pp. 993-1002, 2015.

[73] D. Dhawan, J. A. Ramos-Vara, N. M. Hahn et al., "DNMT1: an emerging target in the treatment of invasive urinary bladder cancer," Urologic Oncology, vol. 31, no. 8, pp. 1761-1769, 2013.

[74] N. M. Hahn, P. L. Bonney, D. Dhawan et al., "Subcutaneous 5 -azacitidine treatment of naturally occurring canine urothelial carcinoma: a novel epigenetic approach to human urothelial carcinoma drug development," The Journal of Urology, vol. 187, no. 1, pp. 302-309, 2012.

[75] A. L. Dill, D. R. Ifa, N. E. Manicke et al., "Lipid profiles of canine invasive transitional cell carcinoma of the urinary bladder and adjacent normal tissue by desorption electrospray ionization imaging mass spectrometry," Analytical Chemistry, vol. 81, no. 21, pp. 8758-8764, 2009.

[76] B. W. Davis and E. A. Ostrander, "Domestic dogs and cancer research: a breed-based genomics approach," ILAR Journal, vol. 55, no. 1, pp. 59-68, 2014.

[77] K. Hanazono, S. Fukumoto, Y. Kawamura, T. Kadosawa, H. Iwano, and T. Uchide, "Epidermal growth factor receptor expression in canine transitional cell carcinoma," The Journal of Veterinary Medical Science, vol. 77, no. 1, pp. 1-6, 2015.

[78] R. L. Vinall, M. S. Kent, and R. W. de Vere White, "Expression of microRNAs in urinary bladder samples obtained from dogs with grossly normal bladders, inflammatory bladder disease, or transitional cell carcinoma," American Journal of Veterinary Research, vol. 73, no. 10, pp. 1626-1633, 2012.

[79] D. Dhawan, J. A. Ramos-Vara, J. C. Stewart, R. Zheng, and D. W. Knapp, "Canine invasive transitional cell carcinoma cell lines: in vitro tools to complement a relevant animal model of invasive urinary bladder cancer," Urologic Oncology, vol. 27, no. 3, pp. 284-292, 2009.

[80] K. Rathore and M. Cekanova, "Animal model of naturally occurring bladder cancer: characterization of four new canine transitional cell carcinoma cell lines," BMC Cancer, vol. 14, no. 1, p. $465,2014$.
[81] A. Chaux, J. S. Cohen, L. Schultz et al., "High epidermal growth factor receptor immunohistochemical expression in urothelial carcinoma of the bladder is not associated with EGFR mutations in exons 19 and 21: a study using formalin-fixed, paraffin-embedded archival tissues," Human Pathology, vol. 43, no. 10, pp. 1590-1595, 2012.

[82] J. S. Ross, K. Wang, R. N. Al-Rohil et al., "Advanced urothelial carcinoma: next-generation sequencing reveals diverse genomic alterations and targets of therapy," Modern Pathology, vol. 27, no. 2, pp. 271-280, 2014.

[83] S. M. Bilgrami, S. A. Qureshi, S. Pervez, and F. Abbas, "Promoter hypermethylation of tumor suppressor genes correlates with tumor grade and invasiveness in patients with urothelial bladder cancer," Spring, vol. 3, no. 1, p. 178, 2014.

[84] K. L. Yap, K. Kiyotani, K. Tamura et al., "Whole-exome sequencing of muscle-invasive bladder cancer identifies recurrent mutations of UNC5C and prognostic importance of DNA repair gene mutations on survival," Clinical Cancer Research, vol. 20, no. 24, pp. 6605-6617, 2014.

[85] F. Koga, S. Yoshida, M. Tatokoro et al., "ErbB2 and $\mathrm{NF} \kappa \mathrm{B}$ overexpression as predictors of chemoradiation resistance and putative targets to overcome resistance in muscle-invasive bladder cancer," PloS One, vol. 6, no. 11, article e27616, 2011.

[86] H. Mochizuki, S. G. Shapiro, and M. Breen, "Detection of BRAF mutation in urine DNA as a molecular diagnostic for canine urothelial and prostatic carcinoma," PloS One, vol. 10, no. 12, article e0144170, 2015.

[87] H. Mochizuki, K. Kennedy, S. G. Shapiro, and M. Breen, "BRAF mutations in canine cancers," PloS One, vol. 10, no. 6, article e0129534, 2015.

[88] C. Saba, M. Paoloni, and C. Mazcko, "A comparative oncology study of iniparib defines its pharmacokinetic profile and biological activity in a naturally-occurring canine cancer model," PloS One, vol. 11, no. 2, article e0149194, 2016.

[89] A. K. LeBlanc, M. Breen, P. Choyke et al., "Perspectives from man's best friend: National Academy of Medicine's Workshop on Comparative Oncology," Science Translational Medicine, vol. 8, no. 324, p. 324ps5, 2016.

[90] National Cancer Institute, "Comparative Oncology Program,” 2016, http://ccr.nci.nih.gov.

[91] S. K. McMillan, P. Boria, G. E. Moore, W. R. Widmer, P. L. Bonney, and D. W. Knapp, "Antitumor effects of deracoxib treatment in 26 dogs with transitional cell carcinoma of the urinary bladder," Journal of the American Veterinary Medical Association, vol. 239, no. 8, pp. 1084-1089, 2011.

[92] S. I. Mohammed, B. A. Craig, A. J. Mutsaers et al., "Effects of the cyclooxygenase inhibitor, piroxicam, in combination with chemotherapy on tumor response, apoptosis, and angiogenesis in a canine model of human invasive urinary bladder cancer," Molecular Cancer Therapeutics, vol. 2, no. 2, pp. 183-188, 2003.

[93] S. I. Mohammed, P. F. Bennett, B. A. Craig et al., "Effects of the cyclooxygenase inhibitor, piroxicam, on tumor response, apoptosis, and angiogenesis in a canine model of human invasive urinary bladder cancer," Cancer Research, vol. 62, no. 2, pp. 356-358, 2002.

[94] D. Dhawan, B. A. Craig, L. Cheng et al., "Effects of short-term celecoxib treatment in patients with invasive transitional cell carcinoma of the urinary bladder," Molecular Cancer Therapeutics, vol. 9, no. 5, pp. 371-1377, 2010. 
[95] L. T. Glickman, M. Raghavan, D. W. Knapp, P. L. Bonney, and M. H. Dawson, "Herbicide exposure and the risk of transitional cell carcinoma of the urinary bladder in Scottish Terriers," Journal of the American Veterinary Medical Association, vol. 224, no. 8, pp. 1290-1297, 2004.

[96] M. Raghavan, D. W. Knapp, P. L. Bonney, M. H. Dawson, and L. T. Glickman, "Evaluation of the effect of dietary vegetable consumption on reducing risk of transitional cell carcinoma of the urinary bladder in Scottish Terriers," Journal of the American Veterinary Medical Association, vol. 227, no. 1, pp. 94-100, 2005.

[97] D. W. Knapp, W. A. Peer, and A. Conteh, "Detection of herbicides in the urine of pet dogs following home lawn chemical application," The Science of the Total Environment, vol. 456-457, pp. 34-41, 2013.

[98] H. G. Parker, A. L. Shearin, and E. A. Ostrander, "Man's best friend becomes biology's best in show: genome analyses in the domestic dog," Annual Review of Genetics, vol. 44, pp. 309-336, 2010.

[99] P. S. Low, W. A. Henne, and D. D. Doorneweerd, "Discovery and development of folic-acid-based receptor targeting for imaging and therapy of cancer and inflammatory diseases," Accounts of Chemical Research, vol. 41, no. 1, pp. 120-129, 2008.

[100] N. Parker, M. J. Turk, E. Westrick, J. D. Lewis, P. S. Low, and C. P. Leamon, "Folate receptor expression in carcinomas and normal tissues determined by a quantitative radioligand binding assay," Analytical Biochemistry, vol. 338, no. 2, pp. 284-293, 2005.

[101] R. W. Naumann, R. L. Coleman, R. A. Burger et al., "PRECEDENT: a randomized phase II trial comparing vintafolide (EC145) and pegylated liposomal doxorubicin (PLD) in combination versus PLD alone in patients with platinumresistant ovarian cancer," Journal of Clinical Oncology, vol. 31, no. 35, pp. 4400-4406, 2013.

[102] P. M. Lorusso, M. J. Edelman, S. L. Bever et al., "Phase I study of folate conjugate EC145 (vintafolide) in patients with refractory solid tumors," Journal of Clinical Oncology, vol. 30, no. 32, pp. 4011-4016, 2012.

[103] D. Dhawan, J. A. Ramos-Vara, J. F. Naughton et al., "Targeting folate receptors to treat invasive urinary bladder cancer," Cancer Research, vol. 73, no. 2, pp. 875-884, 2013.

[104] S. B. Baylin and P. A. Jones, "A decade of exploring the cancer epigenome - biological and translational implications," Nature Reviews. Cancer, vol. 11, no. 10, pp. 726-734, 2011.

[105] S. B. Baylin and P. A. Jones, "Epigenetic determinants of cancer," Cold Spring Harbor Perspectives in Biology, vol. 8, no. 9, 2016.

[106] H. Li, K. B. Chiappinelli, A. A. Guzzetta et al., "Immune regulation by low doses of the DNA methyltransferase inhibitor 5-azacitidine in common human epithelial cancers," Oncotarget, vol. 5, no. 3, pp. 587-598, 2014.

[107] A. Harb-de la Rosa, M. Acker, R. A. Kumar, and M. Manoharan, "Epigenetics application in the diagnosis and treatment of bladder cancer," The Canadian Journal of Urology, vol. 22, no. 5, pp. 7947-7951, 2015.

[108] C. T. Wu, C. F. Wu, C. H. Lu et al., "Expression and function role of DNA methyltransferase 1 in human bladder cancer," Cancer, vol. 117, no. 22, pp. 5221-5233, 2011.

[109] C. M. Fulkerson, D. Dhawan, D. R. Jones et al., "Pharmacokinetics and toxicity of the novel oral demethylating agent zebularine in laboratory and tumor bearing dogs," Veterinary and Comparative Oncology, vol. 15, no. 1, pp. 226-236, 2017.

[110] J. Zhang, S. Wei, L. Liu et al., "NMR-based metabolomics study of canine bladder cancer," Biochimica et Biophysica Acta, vol. 1822, no. 11, pp. 1807-1814, 2012.

[111] Center for Cancer Research, "Comparative Oncology Trials Consortium," 2016, https://ccrod.cancer.gov/confluence/ display/CCRCOPWeb/Comparative+Oncology+Trials +Consortium.

[112] Center for Cancer Research, "Comparative Oncology Trials Consortium," 2016, https://ccrod.cancer.gov/confluence/ display/CCRCOPWeb/Canine+Comparative+Oncolgy + Genomics+Consortium.

[113] G. Hartley, E. Faulhaber, A. Caldwell et al., "Immune regulation of canine tumour and macrophage PD-L1 expression," Veterinary and Comparative Oncology, 2016, Epub ahead of print.

[114] J. Coy, A. Caldwell, L. Chow, A. Guth, and S. Dow, "PD-1 expression by canine $\mathrm{T}$ cells and functional effects of PD-1 blockade," Veterinary and Comparative Oncology, 2017, Epub ahead of print.

[115] N. Maekawa, S. Konnai, T. Okagawa et al., "Immunohistochemical analysis of PD-L1 expression in canine malignant cancers and PD-1 expression on lymphocytes in canine oral melanoma," PloS One, vol. 11, no. 6, article e0157176, 2016.

[116] M. Tagawa, N. Maekawa, S. Konnai, and S. Takagi, "Evaluation of costimulatory molecules in peripheral blood lymphocytes of canine patients with histiocytic sarcoma," PloS One, vol. 11, no. 2, article e0150030, 2016. 

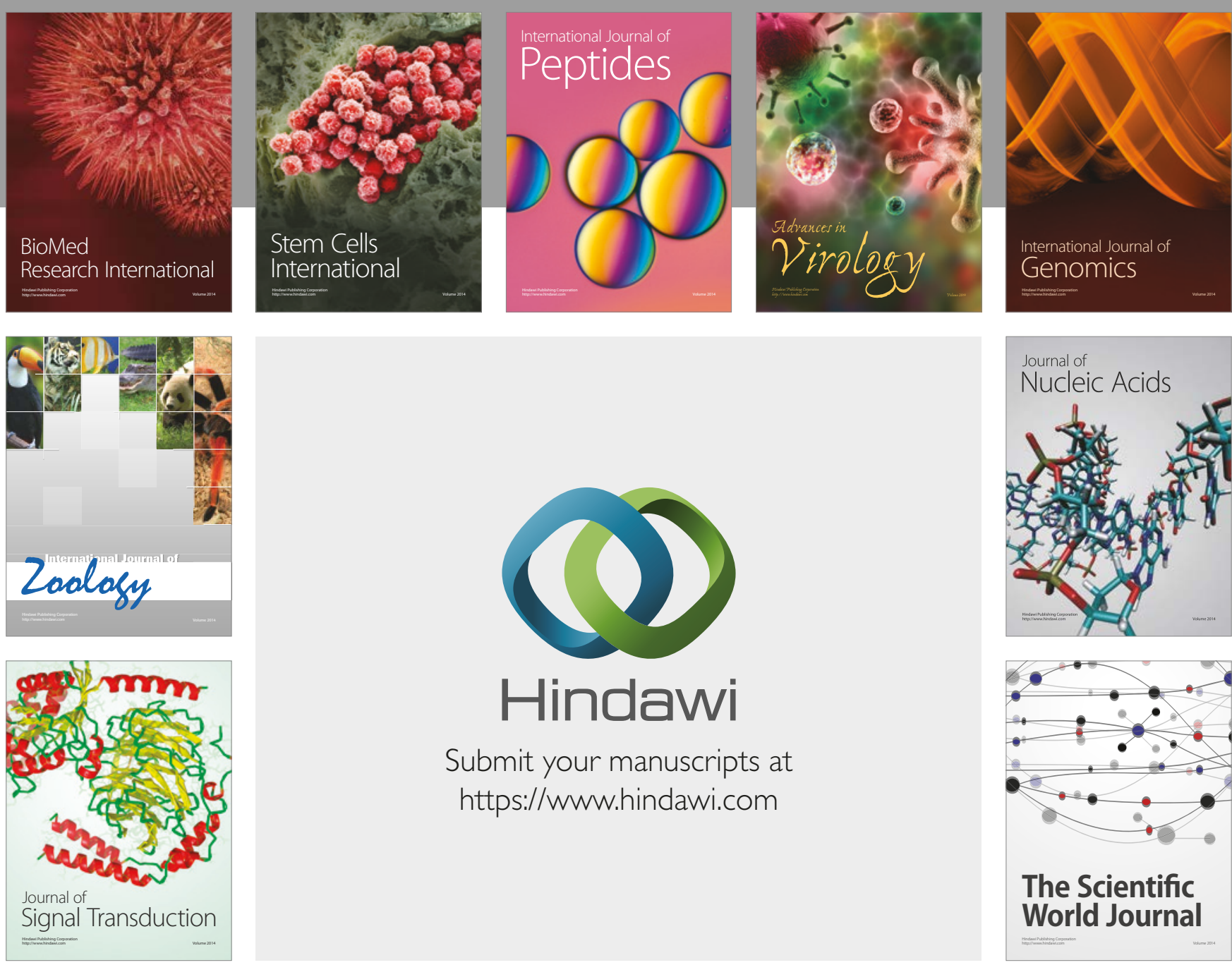

Submit your manuscripts at

https://www.hindawi.com
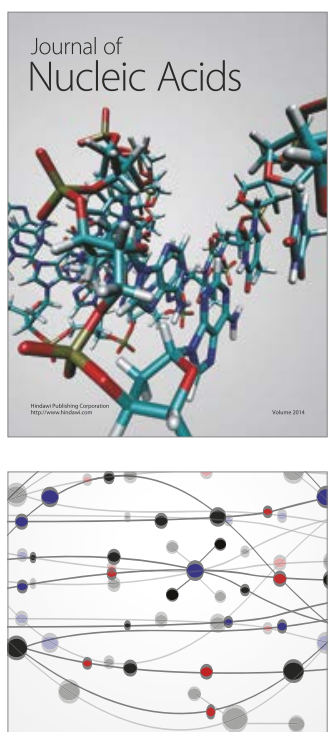

The Scientific World Journal

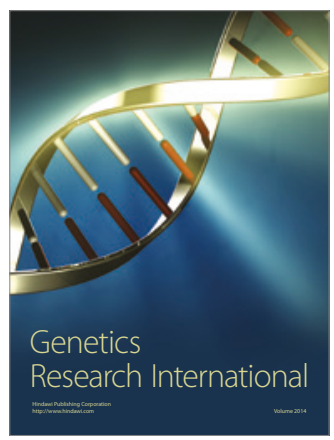

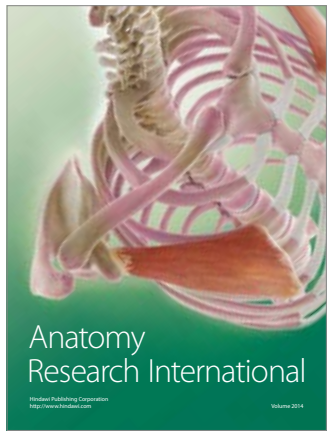

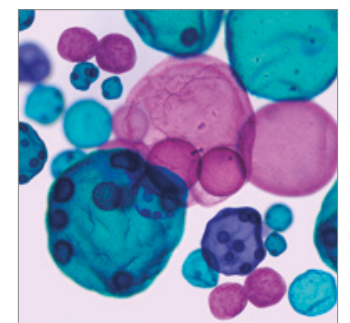

International Journal of Microbiology
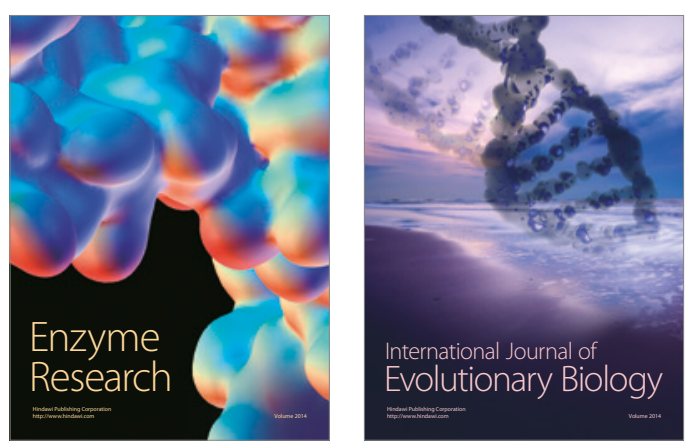
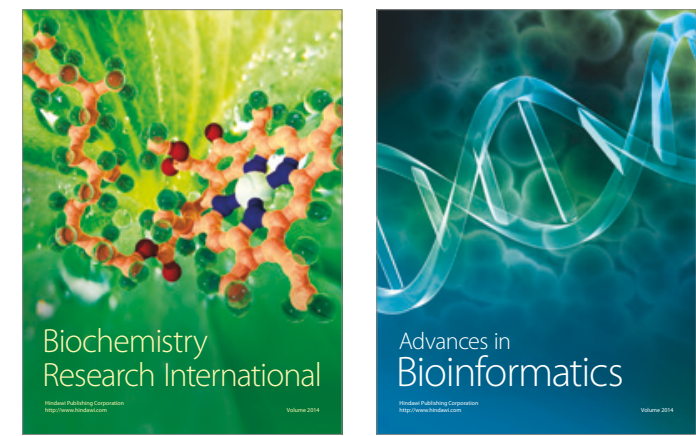

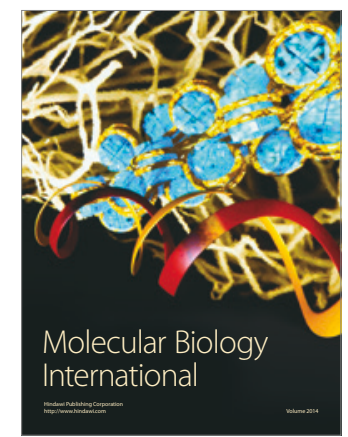

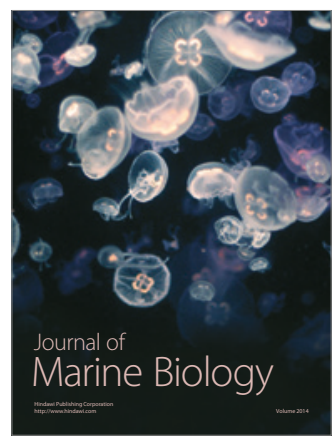

